\title{
Establishment and Characterization of Novel Leukemic Cell Lines Resistant to New-Generation Dihydrofolate Reductase Inhibitor, Pralatrexate
}

\author{
Kana Oiwa, Naoko Hosono, Rie Nishi, Takanori Ueda, Takahiro Yamauchi \\ Department of Hematology and Oncology, University of Fukui, Japan
}

Background: T-cell lymphomas have a poor prognosis due to the chemo-resistant nature of the disease. Pralatrexate $(\mathrm{PDX})$ is a novel dihydrofolate reductase inhibitor with greater affinity for reduced folate carrier (RFC1) and folypolyglutamate synthetase than methotrexate (MTX), resulting in greater internalization and accumulation in tumor cells. In the present study, PDX-resistant leukemic cell lines were established to clarify the precise mechanism of action of PDX and its resistances.

Methods: T-lymphoblastic leukemia cell lines, CEM, MOLT4 and MOLT3, were used. To establish resistant cell lines, the cells were incubated with low-concentrations of PDX or MTX. After repeated passages with escalating concentrations of the drugs, single cell cloning was performed by a limiting dilution method. The $50 \%$ inhibitory concentration (IC50) values of each drug were calculated by XTT assay and the cross-resistances of anti-leukemic agents were also assessed. Cytotoxicity of each drug was evaluated by flow cytometry. The expression levels of RFC1 were assessed by RQ-PCR and Western blotting.

Results: The IC50 values of MTX against CEM, MOLT4 and MOLT3 were $8.2 \mathrm{nM}, 40 \mathrm{nM}$, and $20 \mathrm{nM}$, while the values of PDX were $0.6 \mathrm{nM}, 2.4 \mathrm{nM}$ and $1.4 \mathrm{nM}$, respectively. The corresponding resistant cell lines were 10-50-fold more resistant to MTX or PDX than their parental counterparts. PDX was shown to possess cytotoxicity against MTX-resistant cells, whereas MTX didn't exhibit any cytotoxicity in PDX-resistant cells. After 48 hours of treatment at their IC75 dose, apoptosis was induced in 75.5\% of CEM, 83.2\% of MOLT4, 95.5\% of MOLT3 and 96.1\% of MOLT3/PDX-resistant cells, whereas $10.9 \%$ of CEM/PDX-resistant cells and 16.0\% of MOLT4/PDX-resistant cells were apoptotic. There was no significant difference in RFC1 expression between PDX-resistant cell lines and their parental counterparts. PDXresistant cells were more sensitive to nucleoside analogs than their parental counterparts. There was no cross-resistance to bortezomib or panobinostat in both MTX- and PDX-resistant cells.

Conclusions: We established novel PDX-resistant T-lymphoblastic leukemia cell lines. These cell lines were't induced apoptosis by PDX, but showed high susceptibility to nucleoside analogs. These results may encourage further investigation to overcome resistance of PDX for patients suffering from relapse or refractory T-cell lymphoma. 